\title{
EMF-Aware Cellular Networks in RIS-Assisted Environments
}

\author{
Hussam Ibraiwish, Student Member, IEEE Ahmed Elzanaty, Member, IEEE, Yazan H. \\ Al-Badarneh, Member, IEEE, Mohamed-Slim Alouini, Fellow, IEEE
}

\begin{abstract}
The deployment of the 5th-generation cellular networks (5G) and beyond has triggered health concerns due to the electric and magnetic fields (EMF) exposure. In this paper, we propose a novel architecture to minimize the population exposure to EMF by considering a smart radio environment with a reconfigurable intelligent surface (RIS). Then, we optimize the RIS phases to minimize the exposure in terms of the exposure index (EI) while maintaining a minimum target quality of service. The proposed scheme achieves up to $20 \%$ reduction in EI compared to schemes without RISs.
\end{abstract}

Index Terms-Reconfigurable intelligent surfaces; EMF-aware cellular design; EMF exposure.

\section{INTRODUCTION}

The International Agency on Research on Cancer (IARC) classified radiofrequency radiation (RFR) as possibly carcinogenic to humans (Group 2B) based on experimental studies regarding non-thermal impacts [1], [2]. Recently, the wide deployment of 5th-generation cellular networks $(5 \mathrm{G})$ triggers health concerns among the population regarding electric and magnetic fields (EMF) exposure [3], [4]. Such fear increases for networks that adopt higher frequency bands such as millimeter-waves. In these bands, users have to increase their transmit power to cope with the high path loss, escalating their EMF exposure .

In the literature, some schemes have been proposed to mitigate the EMF exposure. For instance, the authors in [5] design a resource allocation scheme to minimize the EMF exposure while maintaining a minimum quality of service (QoS) for users. In [6], a beamforming based technique is proposed for minimizing the EMF exposure. On the other hand, the authors in [7] consider ferrite meta-material between the mobile phone and human head for reducing the exposure. Recently, unmanned aerial vehicles have been used to reduce the exposure [8].

Most previous works consider legacy networks with only operational optimization such as resource allocation. However, in order to significantly minimize the exposure an architectural development is required, especially for beyond $5 \mathrm{G}$ networks operating at higher frequency bands.

H. Ibraiwish, and M.-S. Alouini are with the Computer, Electrical and Mathematical Sciences and Engineering (CEMSE) Division, King Abdullah University of Science and Technology (KAUST), Thuwal 23955, Saudi Arabia (e-mail: \{hussam.Ibraiwish, slim.alouini\}@kaust.edu.sa).

A. Elzanaty is with the Institute for Communication Systems (ICS), University of Surrey, Guildford GU2 7XH, U.K. (e-mail: a.elzanaty@surrey.ac.uk). He was with CEMSE Division, KAUST.

Y. H. Al-Badarneh is with the Department of Electrical Engineering, The University of Jordan, Amman 11942, Jordan (e-mail: yalbadarneh@ju.edu.jo).

The research has been funded by Competitive Research Grant (CRG) 2020.
Recently, architectural-based solutions exploiting what are called reconfigurable intelligent surfaces (RISs) have been proposed to enhance communication systems performance [9]. Unlike natural surfaces where the incident angle equals to reflected angle, a RIS can reflect the incident wave toward a specified direction depending on the phase shift induced on its surface [10].

In this paper, we propose a novel architecture where a RIS is exploited to minimize the EMF exposure. we consider the uplink (UL) of a cellular network in a RIS assisted environment involving single-antenna mobile users and a multiantenna base station (BS). The contribution of this work can be summarized as follows.

- We propose a novel architecture that exploits a RIS with optimized phase design to minimize the overall exposure by passively focusing users' signals toward the BS to achieve the target data rate with minimal transmit power and exposure.

- We develop a dual gradient descent algorithm to optimize the RIS phases and derive closed-form expressions for the gradient, Hessian matrix, and optimized step size.

For notations, vectors and matrices are denoted by bold small and capital letters, respectively. The $k^{\text {th }}$ column and row of $\mathbf{X}$ are written as $\mathbf{x}_{k}$ and $\mathbf{x}_{(k)}$, respectively, while $x_{m, n}$ represents the element in the $m^{\text {th }}$ row and $n^{\text {th }}$ column. Similarly, $x_{n}$ represents the $n^{\text {th }}$ element of a vector $\mathbf{x}$. The first and second norm of vector $\mathbf{x}$ are represented as $\|\mathbf{x}\|_{1}$ and $\|\mathbf{x}\|$, respectively. The Frobenius norm, pseudo inverse, and Hermitian transpose of $\mathbf{X}$ are represented as $\|\mathbf{X}\|_{\mathrm{F}}, \mathbf{X}^{+}$, and $\mathbf{X}^{\mathrm{H}}$, respectively. The Kronecker product between $\mathbf{X}$ and $\mathbf{Y}$ is denoted by $\mathbf{X} \otimes \mathbf{Y}$. Finally, the real and imaginary part of a complex matrix $\mathbf{Z}$ are denoted by $\mathbf{Z}^{\mathrm{Re}}$ and $\mathbf{Z}^{\mathrm{Im}}$, respectively.

\section{Proposed RIS-ASSISTED ARChitecture}

We consider a single BS, equipped with $M$ antennas, located at $\left(\mathrm{X}^{\mathrm{BS}}, \mathrm{Y}^{\mathrm{BS}}\right)$ serving $K$ users each with a single antenna. A RIS with $N$ reflective elements located at $\left(\mathrm{X}^{\mathrm{RIS}}, \mathrm{Y}^{\mathrm{RIS}}\right)$ is considered to enhance the uplink channel, as in Fig. 11. The received signal at the BS can be written as

$$
\mathbf{y}=\left(\mathbf{H}^{\mathrm{r}} \mathbf{\Phi}(\boldsymbol{\theta}) \mathbf{H}^{\mathrm{u}}+\mathbf{H}^{\mathrm{d}}\right) \mathbf{x}+\mathbf{n},
$$

where $x_{k}$ represents the complex transmitted symbol from user $k$ and $\mathbf{x} \in \mathbb{C}^{K \times 1}$. The transmit power of user $k$ can be simply computed as $p_{k}=\mathbb{E}\left\{x_{k} x_{k}^{*}\right\}$. The matrices $\mathbf{H}^{\mathrm{u}} \in \mathbb{C}^{N \times K}, \mathbf{H}^{\mathrm{r}} \in$ $\mathbb{C}^{M \times N}$, and $\mathbf{H}^{\mathrm{d}} \in \mathbb{C}^{M \times K}$ represent the channel matrix between the users and RIS, RIS and BS, and BS and users, respectively. The element $\theta_{n}$ is the phase shift induced by the $n^{\text {th }}$ element 


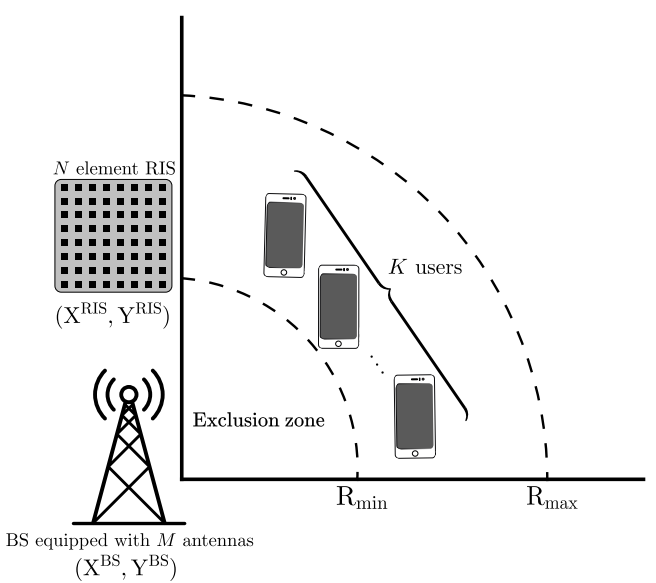

Fig. 1. The system model of the proposed cellular network with a RIS.

of the RIS, $\boldsymbol{\theta} \in \mathbb{R}^{N \times 1}, \phi_{n, n} \triangleq e^{j \theta_{n}}$, and $\boldsymbol{\Phi}(\boldsymbol{\theta}) \in \mathbb{C}^{N \times N}$ is a diagonal matrix. The vector $\mathbf{n} \in \mathbb{C}^{M \times 1}$ represents the additive white Gaussian noise (AWGN) at the BS, where all entries of $\mathbf{n}$ are complex Gaussian random variables with zero means and $\sigma^{2}$ variances. The BS estimates users' signals by applying a linear estimator, i.e., multiplying $\mathbf{y}$ by a beamforming matrix $\mathbf{G} \in \mathbb{C}^{K \times M}$.

\section{EMF-AwARE DESIGN WITH RIS}

A single user exposure can be quantified by the specific absorption rate (SAR), which is defined as the power absorbed per mass of exposed tissue $(\mathrm{W} / \mathrm{Kg})$. The SAR for a certain user can be modeled as a summation of the exposure caused by the cellular phone itself due to the UL, i.e., $\mathrm{SAR}^{\mathrm{UL}}$, and the exposure resulting form the base station due to the downlink (DL), i.e., $\mathrm{SAR}^{\mathrm{DL}}$. The exposure for the $k^{\text {th }}$ user is

$$
\mathrm{SAR}_{k}=\mathrm{SAR}_{k}^{\mathrm{UL}}+\mathrm{SAR}_{k}^{\mathrm{DL}} \triangleq \mathrm{SAR}_{k}^{\mathrm{ref}, \mathrm{UL}} p_{k}+\mathrm{SAR}_{k}^{\mathrm{ref}, \mathrm{DL}} s_{k},
$$

where $p_{k}$ is the transmit power in $\mathrm{W}, \mathrm{SAR}_{k}^{\mathrm{ref}, \mathrm{UL}}$ is the normalized $\mathrm{SAR}_{k}^{\mathrm{UL}}$ (i.e., the induced $\mathrm{SAR}_{k}^{\mathrm{UL}}$ when $p_{k}$ equals $1 \mathrm{~W}), s_{k}$ is the received power density from the BS in W/m $\mathrm{m}^{2}$, and $\mathrm{SAR}_{k}^{\text {ref,DL }}$ is the normalized $\mathrm{SAR}_{k}^{\mathrm{DL}}$ (i.e., $\mathrm{SAR}_{k}^{\mathrm{DL}}$ when $s_{k}$ equals $1 \mathrm{~W} / \mathrm{m}^{2}$ ). To account for the population exposure, we consider the exposure index (EI) metric [11]

$$
\mathrm{EI} \triangleq \sum_{k=1}^{K} \mathrm{SAR}_{k}[\mathrm{~W} / \mathrm{Kg}]
$$

The BS transmit power is significantly higher than that of users. However, the received power density $s_{k}$ can be quite low, because users are usually far from the BS, i.e. located outside the exclusion zone. According to the Federal Communications Commission (FCC), the received power density near typical cellular towers is usually less than $10 \mathrm{~mW} / \mathrm{m}^{2}$. Using a typical value of $\operatorname{SAR}_{k}^{\text {ref,DL }}$ in [11], we have a $\mathrm{SAR}_{k}^{\mathrm{DL}}$ of $42 \mu \mathrm{W} / \mathrm{Kg}$. On the other hand, for the maximum transmit power for a mobile phone of $200 \mathrm{~mW}$, we have $\mathrm{SAR}_{k}^{\mathrm{UL}}=1060 \mu \mathrm{W} / \mathrm{Kg}$, which is significantly higher than the DL exposure, as also shown in [8]. Hence, we consider only the UL exposure. Now, the EI can be written as 1

$$
\mathrm{EI}(\mathbf{p})=\sum_{k=1}^{K} \mathrm{SAR}_{k}^{\mathrm{ref}} p_{k} \quad[\mathrm{~W} / \mathrm{Kg}],
$$

\footnotetext{
${ }^{1}$ Since only the UL exposure is considered, we remove the UL superscript.
}

Then, the optimization problem can be formulated as

$$
\begin{array}{ll}
\underset{\mathbf{p}, \boldsymbol{\theta}, \mathbf{G}}{\operatorname{minimize}} & \mathrm{EI}(\mathbf{p}) \\
\text { subject to } & r_{k}(\boldsymbol{\theta}, \mathbf{G}, \mathbf{p}) \triangleq \log _{2}\left(1+\operatorname{SINR}_{k}\right) \geq r_{k}^{\text {th }} \\
& p_{k} \leq p_{\max }, \forall k \in \mathbb{K} \triangleq\{1,2, \cdots, K\},
\end{array}
$$

where $r_{k}$ in bits/Hz is the actual spectral efficiency for user $k$, $r_{k}^{\text {th }}$ is the minimum spectral efficiency required by user $k, p_{\max }$ is the maximum allowable transmit power of users' devices, which also limits the exposure experienced by each user, and $\mathrm{SINR}_{k}$ is the signal-to-interference plus noise ratio (SINR) of the $k^{\text {th }}$ user. The SINR can be written as

$$
\operatorname{SINR}_{k}=\frac{\left|\mathbf{g}_{(k)}\left(\mathbf{H}^{\mathrm{r}} \boldsymbol{\Phi}(\boldsymbol{\theta}) \mathbf{h}_{k}^{\mathrm{u}}+\mathbf{h}_{k}^{\mathrm{d}}\right) x_{k}\right|^{2}}{\sigma^{2}\left\|\mathbf{g}_{(k)}\right\|^{2}+\sum_{i=1, i \neq k}^{K}\left|\mathbf{g}_{(k)}\left(\mathbf{H}^{\mathrm{r}} \mathbf{\Phi}(\boldsymbol{\theta}) \mathbf{h}_{i}^{\mathrm{u}}+\mathbf{h}_{i}^{\mathrm{d}}\right) x_{i}\right|^{2}},
$$

where $\mathbf{g}_{(k)}$ is the $k^{\text {th }}$ row of the beamforming matrix $\mathbf{G}$.

\section{OPtimization Algorithm}

\section{A. Problem Reduction}

The problem is non-convex and requires joint optimization of the beamforming, users' transmit power, and RIS phases. We propose an alternate optimization algorithm where we first design the beamforming and user allocated power for a fixed RIS phases profile. Then, we optimize the RIS phases and iterate between these two processes till convergence.

For a fixed RIS phase profile, the optimal beamforming vectors and allocated powers for this problem can be obtained as in [12]. Nevertheless, this method requires high computational complexity iterative algorithm. Also, it does not suppress the interference from various users at the BS, further complicating the problem. In this regard, we design the beamformer so that it eliminates the cross-interference from various users at the BS, i.e., zero-forcing (ZF) beamforming. Hence, the beamforming matrix at the BS can be written as [13]

$$
\mathbf{G}(\boldsymbol{\theta})=\left(\mathbf{H}^{\mathrm{r}} \boldsymbol{\Phi}(\boldsymbol{\theta}) \mathbf{H}^{\mathrm{u}}+\mathbf{H}^{\mathrm{d}}\right)^{+}, \quad \text { for } N \geq K \text { and } M \geq K \text {. }
$$

By noting that $\left|\mathbf{g}_{(k)}\left(\mathbf{H}^{\mathrm{r}} \boldsymbol{\Phi}(\boldsymbol{\theta}) \mathbf{h}_{k}^{u}+\mathbf{h}_{k}^{d}\right) x_{k}\right|^{2}=p_{k}$ and $\sum_{i=1, i \neq k}^{K}\left|\mathbf{g}_{(k)}\left(\mathbf{H}^{\mathrm{r}} \boldsymbol{\Phi}(\boldsymbol{\theta}) \mathbf{h}_{i}^{\mathrm{u}}+\mathbf{h}_{i}^{\mathrm{d}}\right) x_{i}\right|^{2}=0$, the SINR can be written as $\operatorname{SINR}_{k}=p_{k} \sigma^{-2}\left\|\mathbf{g}_{(k)}\right\|^{-2}$.

Since the objective function and achieved data rate are monotonically increasing in the users' transmit power, the optimal $p_{k}$ is the power that achieves the minimum required data rate $r_{k}^{\text {th }}$. Otherwise, this will lead to an unnecessary exposure rise. Thus, the power of the $k^{\text {th }}$ user is set to

$$
p_{k}(\boldsymbol{\theta})=\left(2^{r_{k}^{\mathrm{th}}}-1\right) \sigma^{2}\left\|\mathbf{g}_{(k)}\right\|^{2} \triangleq \widetilde{\sigma}_{k}^{2}\left\|\mathbf{g}_{(k)}\right\|^{2}, \forall k \in \mathbb{K} .
$$

Accordingly, we can eliminate the data rate constraint by substituting (8) in (5a). After representing both $\mathbf{G}$ and $\mathbf{p}$ as functions of $\boldsymbol{\theta}$ as in (7) and (8), the optimization problem is relaxed to

$$
\begin{array}{ll}
\underset{\boldsymbol{\theta}}{\operatorname{minimize}} & \sum_{k=1}^{K} \operatorname{SAR}_{k}^{\mathrm{ref}} \widetilde{\sigma}_{k}^{2}\left\|\mathbf{g}_{(k)}\right\|^{2} \\
\text { subject to } & \widetilde{\sigma}_{k}^{2}\left\|\mathbf{g}_{(k)}\right\|^{2} \leq p_{\max } .
\end{array}
$$


Although problem (9) is not identically equivalent to (5), it can lead to a solution that sufficiently reduces the EI with low computational complexity.

As we can see in (8), larger $\widetilde{\sigma}_{k}^{2}$ values necessitate higher power to be transmitted from the mobile and possibly that power will exceed $p_{\max }$. Hence, the maximum $p_{k}$ is forced to be $p_{\max }$, which can result in a rate, $r_{k}$, that is less than the target rate, $r_{k}^{\text {th }}$, i.e., the problem becomes infeasible. Therefore, we define the rate satisfaction ratio for the users as $\sum_{k=1}^{K} r_{k} / \sum_{k=1}^{K} r_{k}^{\text {th }}$, quantifying the solution feasibility. Even though the problem is still non-convex, applying various convex optimization algorithms can significantly reduce the objective function, leading to a local minimum. The solution for the above problem must satisfies the Karush-Kuhn-Tucker (KKT) conditions where it must be a saddle point within the Lagrangian function. Thus, our new objective is to find a saddle point for the following Lagrangian function

$$
\mathcal{L}(\boldsymbol{\theta}, \lambda)=\operatorname{EI}(\mathbf{p}(\boldsymbol{\theta}))+\lambda^{\top}\left(\mathbf{p}(\boldsymbol{\theta})-\mathbf{p}_{\max }\right),
$$

where $\lambda \in \mathbb{R}^{K \times 1}$ represents the KKT multipliers and $\mathbf{p}_{\max } \in$ $\mathbb{R}^{K \times 1}$ is a vector with each entity being $p_{\max }$.

In this work, we propose the dual gradient descent algorithm where we iteratively apply two phases [14]. In the first phase, the Lagrangian function is minimized by applying the gradient descent considering only the optimization variables while in the second phase, the Lagrangian function is maximized by applying the gradient ascent considering only the multipliers, thus both gradient descent and ascent are adopted. At the $t^{\text {th }}$ iteration, the algorithm updates the phases and multipliers as

$$
\begin{aligned}
& \boldsymbol{\theta}^{\{t\}}=\boldsymbol{\theta}^{\{t-1\}}-\alpha \nabla_{\boldsymbol{\theta}} \mathcal{L}\left(\boldsymbol{\theta}^{\{t-1\}}, \lambda^{\{t-1\}}\right) \\
& \lambda^{\{t\}}=\lambda^{\{t-1\}}+\beta \nabla_{\lambda} \mathcal{L}\left(\boldsymbol{\theta}^{\{t\}}, \lambda^{\{t-1\}}\right),
\end{aligned}
$$

where $\alpha$ and $\beta$ are the step sizes for updating the RIS phases and multipliers, respectively. The gradient of the Lagrangian function with respect to both of the RIS phases and KKT multipliers and the step sizes are investigated below.

\section{B. Gradient of the Lagrangian Function}

Let $a_{k} \triangleq\left(\mathrm{SAR}_{k}^{\mathrm{ref}}+\lambda_{k}\right) \widetilde{\sigma}_{k}^{2}, \mathbf{A} \triangleq \operatorname{diag}\left[a_{1}, a_{2}, \ldots, a_{k}\right], \overline{\mathbf{H}}^{\mathrm{u}} \triangleq$ $\mathbf{H}^{\mathrm{u}} \sqrt{\mathbf{A}^{-1}}$, and $\overline{\mathbf{H}}^{\mathrm{d}} \triangleq \mathbf{H}^{\mathrm{d}} \sqrt{\mathbf{A}^{-1}}$, the Lagrangian function in 10 ) can be simplified as

$$
\mathcal{L}(\boldsymbol{\theta}, \boldsymbol{\lambda})=\operatorname{tr}(\mathbf{T})-p_{\max }\|\lambda\|_{1},
$$

where $\mathbf{T} \triangleq\left(\mathbf{Q}^{H} \mathbf{Q}\right)^{-1}$ and $\mathbf{Q} \triangleq \mathbf{H}^{\mathrm{r}} \mathbf{\Phi} \overline{\mathbf{H}}^{\mathrm{u}}+\overline{\mathbf{H}}^{\mathrm{d}}$. The derivative of the Lagrangian function with respect to $\theta_{i}$ is denoted by $\mathcal{L}^{\prime}(i)$ and can be derived using matrix calculus as ${ }^{2}$

$$
\mathcal{L}^{\prime}(i) \triangleq \frac{\partial \mathcal{L}}{\partial \theta_{i}}=\Re\left\{\operatorname{tr}\left(j e^{j \theta_{n}}\left(\mathbf{h}_{i}^{\mathrm{r}} \otimes \overline{\mathbf{h}}_{(i)}^{\mathrm{u}}\right)\left(\frac{\partial \mathcal{L}}{\partial \mathbf{Q}}\right)^{H}\right)\right\},
$$

where

$$
\frac{\partial \mathcal{L}}{\partial \mathbf{Q}}=-2 \mathbf{Q} \mathbf{T}^{2},
$$

as shown in the supplemental material and in the extended version [15]. On the other hand, the derivative with respect to the multipliers can be written as

$$
\frac{\partial \mathcal{L}}{\partial \lambda_{i}}=\widetilde{\sigma}_{k}^{2}\left\|\mathbf{g}_{(i)}\right\|^{2}-p_{\max }, \quad \forall i \in\{1,2, \cdots, N\} .
$$

\footnotetext{
${ }^{2}$ For notational simplicity, we drop the dependency on $\boldsymbol{\theta}$ and $\lambda$ in $\mathcal{L}(\boldsymbol{\theta}, \lambda)$.
}

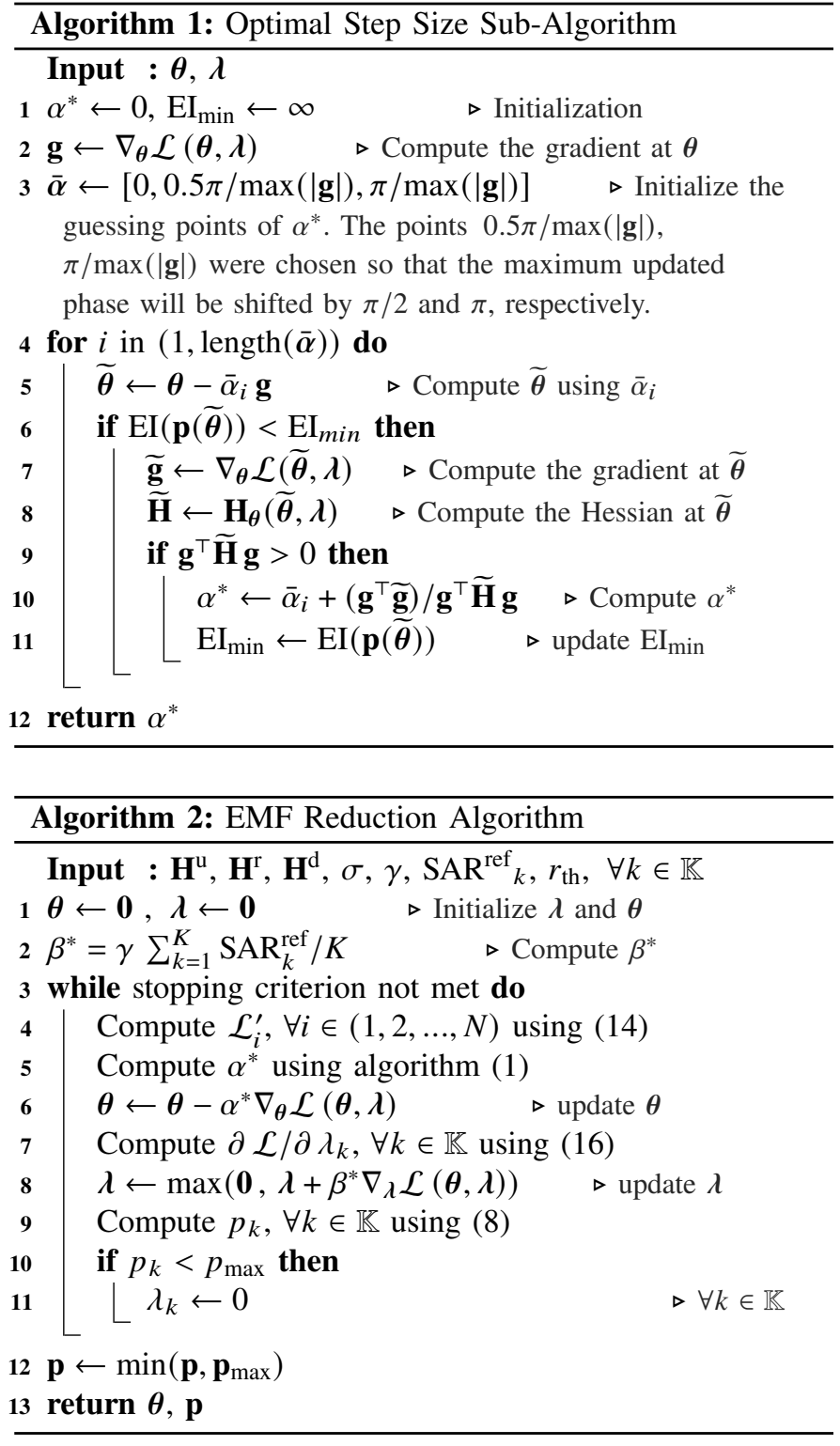

\section{Optimal Step Size}

Regarding the step size for the phase update, we approximate the Lagrangian function around an initial step size $\bar{\alpha}$ by a quadratic function. Then, the optimal step size $\alpha^{*}$ of the approximated Lagrangian function can be found as

$$
\alpha^{*}=\bar{\alpha}+\frac{\left(\nabla_{\boldsymbol{\theta}} \mathcal{L}(\boldsymbol{\theta}, \lambda)\right)^{\top} \nabla_{\boldsymbol{\theta}} \mathcal{L}(\widetilde{\boldsymbol{\theta}}, \lambda)}{\left(\nabla_{\boldsymbol{\theta}} \mathcal{L}(\boldsymbol{\theta}, \lambda)\right)^{\top} \mathbf{H}_{\boldsymbol{\theta}}(\widetilde{\boldsymbol{\theta}}, \lambda) \nabla_{\boldsymbol{\theta}} \mathcal{L}(\boldsymbol{\theta}, \lambda)},
$$

where $\widetilde{\boldsymbol{\theta}}=\boldsymbol{\theta}-\bar{\alpha} \nabla_{\boldsymbol{\theta}} \mathcal{L}(\boldsymbol{\theta}, \boldsymbol{\lambda})$ and $\mathbf{H}_{\boldsymbol{\theta}}(\widetilde{\boldsymbol{\theta}}, \boldsymbol{\lambda}) \in \mathbb{R}^{N \times N}$ is the Hessian matrix of the Lagrangian function computed at $\widetilde{\boldsymbol{\theta}}$ and $\lambda$. The elements of the Hessian matrix can be computed as

$$
\frac{\partial^{2} \mathcal{L}}{\partial \theta_{v} \partial \theta_{u}}=\left\{\begin{array}{ll}
\Re\left\{\psi_{v, u}\right\} & , v \neq u \\
\Re\left\{\operatorname{tr}\left(j \mathbf{R T}^{2} \mathbf{Q}^{H}\right)+\psi_{v, v}\right\} & , v=u
\end{array},\right.
$$

where

$$
\begin{aligned}
\psi_{v, u} & \triangleq \operatorname{tr}\left(j e^{j \theta_{u}}\left(\mathbf{h}_{u}^{\mathrm{r}} \otimes \overline{\mathbf{h}}_{(u)}^{\mathrm{u}}\right)\left(\frac{\partial \mathcal{L}^{\prime}(v)}{\partial \mathbf{Q}}\right)^{H}\right), \\
\frac{\mathcal{L}^{\prime}(v)}{\partial \mathbf{Q}} & =\mathbf{R T}^{2}-\mathbf{Q T}(\mathbf{T C}-\mathbf{C T}) \mathbf{T},
\end{aligned}
$$




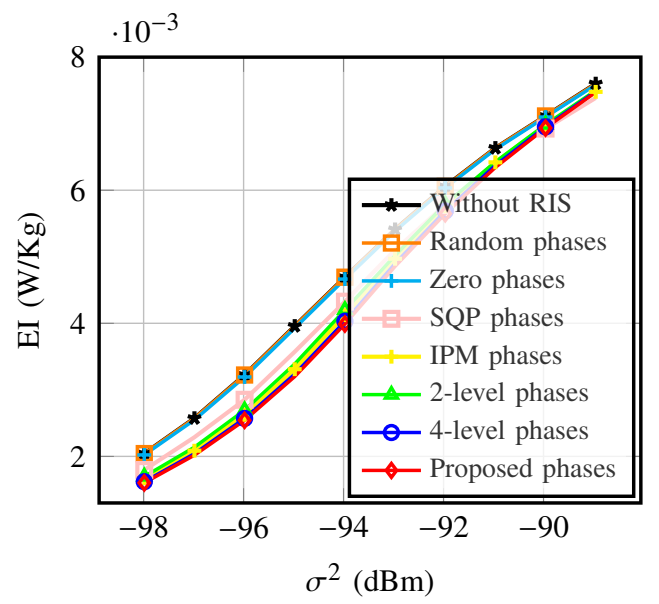

Fig. 2. The EI vs. $\sigma^{2}$ considering different scenarios, for $K=16, N=128$, and $M=32$.

for $\mathbf{R} \triangleq-2 j e^{j \theta_{v}}\left(\mathbf{h}_{v}^{\mathrm{r}} \otimes \overline{\mathbf{h}}_{(v)}^{\mathrm{u}}\right)$ and $\mathbf{C} \triangleq \mathbf{R}^{H} \mathbf{Q}-\mathbf{Q}^{H} \mathbf{R}$.

The optimal step size in (17) is computed considering a given initial step size. Algorithm 1 identifies the procedure required to obtain an appropriate step size that minimizes the Lagrangian function. More precisely, the proposed algorithm begins with three initial step sizes, then the one corresponding to the minimum EI and positive curvature is chosen. In rare situations when all the initial guesses have a negative curvature, the phases will not be updated, i.e., $\alpha^{*}=0$, and the main algorithm will terminate.

On the other hand, reasonable values for the step size of the multipliers, $\beta$, is on the order of the average reference SAR. In particular, we set $\beta=\gamma \sum_{k=1}^{K} \mathrm{SAR}_{k}^{\text {ref }} / K$, where $\gamma$ is a small constant around unity that determines how quickly the KKT multipliers should be updated. For small values of $\gamma$ (i.e., small multipliers), the Lagrangian will be closer to the main objective function, yielding smaller EI, albeit with lower rate satisfaction ratio. On the other hand, larger $\gamma$ results in more feasible users. Thus, there is a trade off between the EI and rate satisfaction ratio depending on the choice of $\gamma$.

\section{Complexity Analysis}

The computational complexity of computing the gradient is $O(N M K)$, while the computational complexity of computing the Hessian is $O\left(N^{2} M K\right)$. Thus, our algorithm is overwhelmed by computing the Hessian matrix which is essential to compute the optimal step size. Therefore, the overall complexity of our algorithm is $O\left(N^{2} M K\right)$. All the computations are performed by the BS, where it starts by estimating $\mathbf{H}^{\mathrm{u}}, \mathbf{H}^{\mathrm{r}}$ and $\mathbf{H}^{\mathrm{d}}$, then it optimizes the RIS phases and feeds such information back to the RIS 3

\section{Numerical Results}

In this section, we investigate the performance of our algorithm. We set the environment as shown in Fig. 1. where there are $K=16$ active users uniformly distributed in the shown area. We set $R_{\min }$ and $R_{\max }$ to $10 \mathrm{~m}$ and $150 \mathrm{~m}$, respectively. The BS and RIS are at a height of $10 \mathrm{~m}$ and located at $(0,0)$ and $(30,20) \mathrm{m}$, respectively. We consider a Rician fading for the RIS-users and RIS-BS links, as the paths involving RIS usually have a line-of-sight (LoS) component.

\footnotetext{
${ }^{3}$ For instance, the channels $\mathbf{H}^{\mathrm{u}}, \mathbf{H}^{\mathrm{r}}$ and $\mathbf{H}^{\mathrm{d}}$ can be estimated using various methods as shown in $[16]$ and the references therein.
}

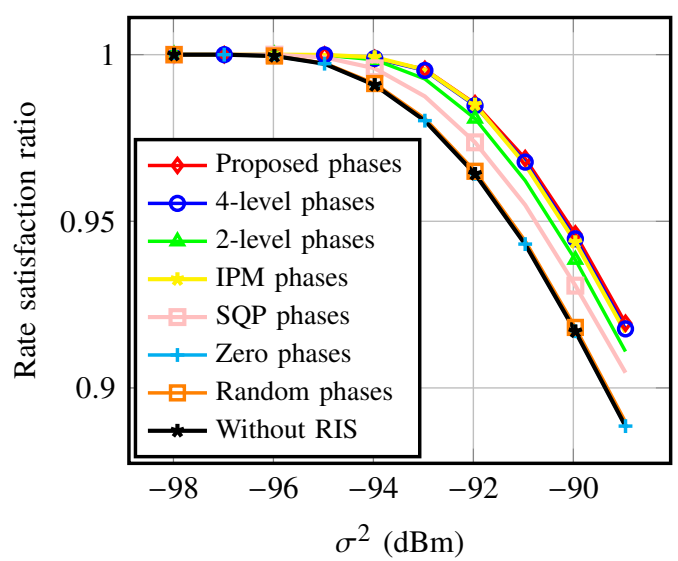

Fig. 3. Rate satisfaction ratio vs. $\sigma^{2}$ considering different scenarios, for $K=16, N=128$, and $M=32$.

Rayleigh fading is considered for user-BS links. In particular, we have

$$
\begin{aligned}
& \mathbf{H}^{\mathrm{r}}=\operatorname{PL}_{\mathrm{LOS}}\left(d^{\mathrm{RB}}\right)\left(\sqrt{\frac{\kappa}{\kappa+1}} \mathbf{a}_{N}^{\top} \mathbf{a}_{M}+\sqrt{\frac{1}{\kappa+1}} \tilde{\mathbf{H}}^{\mathrm{r}}\right), \\
& \mathbf{h}_{k}^{\mathrm{u}}=\operatorname{PL}_{\mathrm{LOS}}\left(d_{k}^{\mathrm{UR}}\right)\left(\sqrt{\frac{\kappa}{\kappa+1}} \mathbf{a}_{k}+\sqrt{\frac{1}{\kappa+1}} \tilde{\mathbf{h}}_{k}^{\tilde{\mathrm{u}}}\right), \\
& \mathbf{h}_{k}^{\mathrm{d}}=\operatorname{PL}_{\mathrm{NLOS}}\left(d^{\mathrm{U}_{\mathrm{k}} \mathrm{B}}\right) \tilde{\mathbf{h}}_{k}^{\tilde{\mathrm{u}}},
\end{aligned}
$$

where $\mathbf{h}_{k}^{\mathrm{u}}$ and $\mathbf{h}_{k}^{\mathrm{d}}$ are the $k^{\text {th }}$ columns of the matrices $\mathbf{H}^{\mathrm{u}}$ and $\mathbf{H}^{\mathrm{d}}$, respectively, $d^{\mathrm{RB}}$ is the distance between the RIS and the BS, $d_{k}^{\mathrm{UR}}$ is the distance between the $k^{\text {th }}$ user and RIS, $d_{k}^{\mathrm{UB}}$ is the distance between the $k^{\text {th }}$ user and the BS, and $\kappa$ is the Rician factor which is set to 10 in all simulations. The functions $\mathrm{PL}_{\mathrm{LOS}}(d)$ and $\mathrm{PL}_{\mathrm{NLOS}}(d)$ are the path losses at distance $d$ for LoS and non-line-of-sight (NLoS) links, respectively. Both $\mathrm{PL}_{\mathrm{LOS}}(d)$ and $\mathrm{PL}_{\mathrm{NLOS}}(d)$ are set according to the 3GPP model [17] as $\operatorname{PL}_{\mathrm{LOS}}(d)=-35.6+22 \log _{10}(d)(\mathrm{dB})$ and $\operatorname{PL}_{\mathrm{NLOS}}(d)=32.6+36.7 \log _{10}(d)(\mathrm{dB})$. All the elements of $\tilde{\mathbf{H}^{\mathrm{r}}}, \tilde{\mathbf{h}}_{k}^{\tilde{\mathrm{u}}}$ and $\tilde{\mathbf{h}_{k}^{\tilde{\mathrm{u}}}}$ are independent, identically distributed (i.i.d.) complex Gaussian random variables with zero mean and unit variance. Finally, $\mathbf{a}_{M}, \mathbf{a}_{N}$ and $\mathbf{a}_{k}$ are the steering vectors [18. (2)].

In these simulations, each user is generated with a $75 \%$ probability of being a data user and a $25 \%$ probability of being a voice user. Each data user has a minimum data rate of $600 \mathrm{Mb} / \mathrm{s}$ and an allocated bandwidth of $100 \mathrm{MHz}$. Each voice user has a minimum data rate of $13.3 \mathrm{~Kb} / \mathrm{s}$ and an allocated bandwidth of $7 \mathrm{KHz}$. The reference SAR can be found from [11, Table 27] by averaging over different postures and population ages. The data users are assumed to use a mobile from a small distance in front of their torso, while voice users are assumed to put the mobile near their head resulting in $\mathrm{SAR}^{\mathrm{ref}}=41 \times 10^{-4}$ and $\mathrm{SAR}^{\mathrm{ref}}=63 \times 10^{-4} \mathrm{~W} / \mathrm{Kg}$ per unit power, respectively.

\section{A. Effect of RIS Phase Design}

We compare the performance of our proposed algorithm in terms of the EI and satisfied rate ratio with schemes without RIS and with various RIS phase profiles, i.e., (i) zero phases with $\boldsymbol{\theta}=\mathbf{0}$; (ii) random phases, where the phase shifts are uniformly distributed from 0 to $2 \pi$; (iii) numerically optimized phases through the interior-point method (IPM) and sequential quadratic programming (SQP). (iv) $L$-level phases where the 


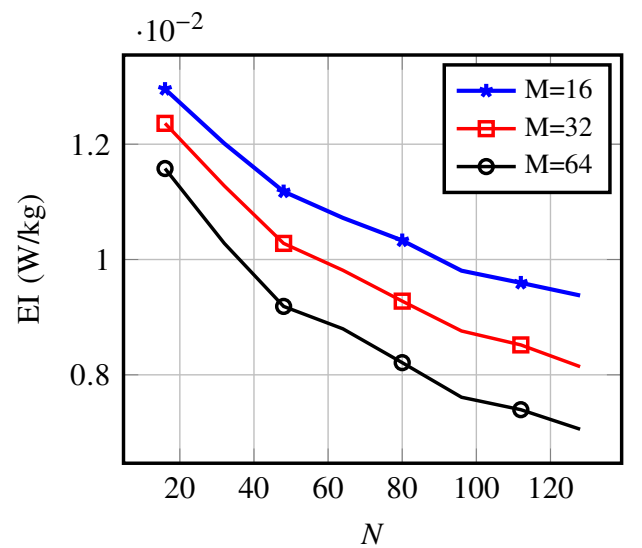

Fig. 4. The EI vs. the number of RIS elements for different number of BS antennas with $K=16$.

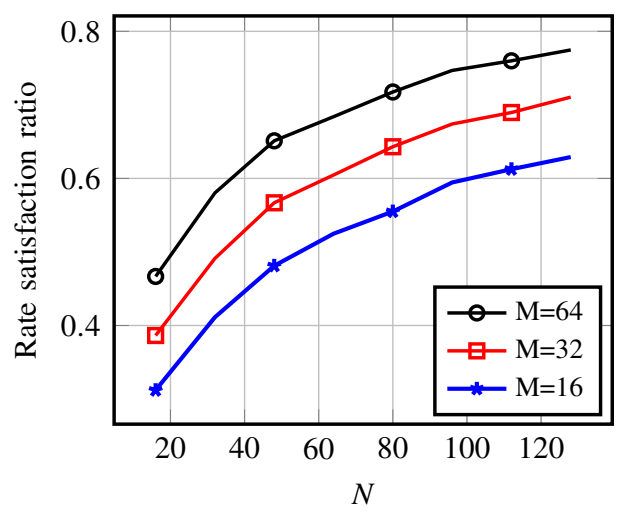

Fig. 5. Rate satisfaction ratio vs. the number of RIS elements for different number of BS antennas with $K=16$.

optimized RIS phases are discretized with $L$ levels uniform quantizer. In Fig 2, the EI for various noise levels is shown for different configurations. We notice that exploiting the RIS with optimized phases reduces the EI by $20 \%$ compared to the schemes without RIS and with non-optimized phases. Also, the EI with random phases coincides with zero phases, because the EI is averaged over a large number of channel realizations. Finally, increasing the number of quantization levels yields results closer to the optimal solution. The data rate satisfaction ratio is shown in Fig. 3 The optimized phases design results in higher rate satisfaction ratio, and consequently higher achieved data rate, compared to other schemes.

\section{B. Effect of Number of RIS Elements}

Here, we see how the number of RIS elements $N$ affects the EI and rate satisfaction ratio for different number of BS antennas, as shown in Fig. 4 and Fig. 5 , respectively. We can see that increasing $N$ or $M$ always yields a better performance in terms of the EI and the rate satisfaction ratio.

\section{Algorithm Convergence}

The convergence of the dual gradient descent algorithm to a global minimum for convex optimization problems is discussed in [14]. Although the problem is non convex the algorithm converges to a local minimum, as shown in Fig. 6 for different noise power values.

\section{CONCLUSION}

We utilized a RIS to minimize the users' exposure to EMF for beyond $5 \mathrm{G}$ networks. We proposed a novel algorithm for minimizing the population UL exposure in terms of the

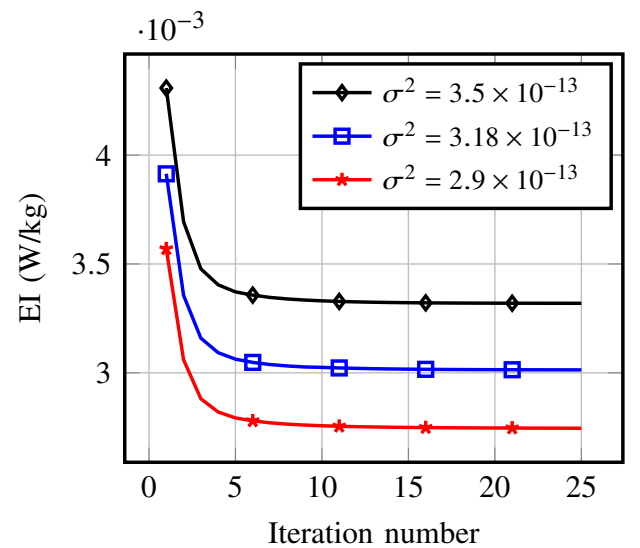

Fig. 6. The EI vs. iteration number, for $K=16, N=128$, and $M=32$.

EI while maintaining a target data rate. For instance, the proposed algorithm achieved a $20 \%$ reduction in EI compared to the schemes without RIS and with non-optimized phases. Furthermore, the optimized phase design achieved higher UL data rates, even at harsh channel conditions.

\section{REFERENCES}

[1] "IARC classifies radiofrequency electromagnetic fields as possibly carcinogenic to humans," IARC and others, Lyon, France, Press release 208, May 2011.

[2] National Toxicology Program, "Toxicology and carcinogenesis studies in Hsd: Sprague dawley SD rats exposed to whole-body radio frequency radiation at a frequency $(900 \mathrm{MHz})$ and modulations (GSM and CDMA) used by cell phones," NTO, Tech. Rep., 2018.

[3] L. Chiaraviglio, A. Elzanaty, and M.-S. Alouini, "Health risks associated with 5G exposure: A view from the communications engineering perspective," IEEE OJ-COMS, vol. 2, pp. 2131-2179, 2021.

[4] A. Elzanaty, L. Chiaraviglio, and M.-S. Alouini, "5G and EMF exposure: Misinformation, open questions, and potential solutions," Front. Comms. Net., vol. 2, p. 5, 2021.

[5] Y. Sambo, M. Al-Imari, F. Héliot, and M. Imran, "Electromagnetic emission-aware schedulers for the uplink of OFDM wireless communication systems," IEEE Trans. Veh. Technol., vol. 66, no. 2, pp. 13131323, 2017.

[6] M. Wang et al., "Evaluation and optimization of the specific absorption rate for multiantenna systems," IEEE Trans. on Electromag. Compat., vol. 53, no. 3, pp. 628-637, 2011.

[7] Y. A. Sambo, F. Héliot, and M. A. Imran, "A survey and tutorial of electromagnetic radiation and reduction in mobile communication systems," IEEE Commun. Surv. Tutor., vol. 17, no. 2, pp. 790-802, 2015.

[8] Z. Lou, A. Elzanaty, and M.-S. Alouini, "Green tethered UAVs for EMFaware cellular networks," IEEE Trans. Green Commun. Netw., 2021.

[9] E. Basar, M. Di Renzo, J. De Rosny, M. Debbah, M.-S. Alouini, and R. Zhang, "Wireless communications through reconfigurable intelligent surfaces," IEEE Access, vol. 7, pp. 116 753-116773, 2019.

[10] M. Najafi, V. Jamali, R. Schober, and H. V. Poor, "Physics-based modeling and scalable optimization of large intelligent reflecting surfaces," IEEE Trans. Commun., vol. 69, no. 4, pp. 2673-2691, 2020.

[11] G. Vermeeren et al., "Low EMF exposure future networks D2. 8 global wireless exposure metric definition," Tech. Rep., 2015.

[12] B. Song, R. L. Cruz, and B. D. Rao, "Network duality for multiuser MIMO beamforming networks and applications," IEEE Trans. Commun., vol. 55, no. 3, pp. 618-630, 2007.

[13] H. Q. Ngo, Massive MIMO: Fundamentals and system designs. Linköping University Electronic Press, 2015, vol. 1642.

[14] R. Tibshirani. Dual ascent. Last Accessed, 20th Jan. 2021. [Online]. Available: https://www.stat.cmu.edu/ ryantibs/convexopt-F18/lectures/ dual-ascent.pdf

[15] H. Ibraiwish, A. Elzanaty, Y. Al-Badarneh, and M.-S. Alouini, "EMFaware cellular networks in RIS-assisted environments," arXiv preprint, 2021.

[16] X. Wei, D. Shen, and L. Dai, "Channel estimation for RIS assisted wireless communications-part I: Fundamentals, solutions, and future opportunities," IEEE Commun. Lett, vol. 25, no. 5, pp. 1398-1402, 2021.

[17] "Evolved universal terrestrial radio access (E-UTRA); further advancements for E-UTRA physical layer aspects (release 9)," Mar. 2010.

[18] S. Cho, H. Song, K.-J. You, and H.-C. Shin, "A new direction-of-arrival estimation method using automotive radar sensor arrays," Int. J. Distrib. Sens. Netw., vol. 13, no. 6, 2017. 